\title{
Perturbations in electromagnetic dark energy
}

\author{
Jose Beltrán Jiménez ${ }^{a}$, Tomi S. Koivisto ${ }^{b}$, Antonio L. Maroto ${ }^{a}$ and David F. Mota ${ }^{c}$ \\ ${ }^{a}$ Departamento de Física Teórica, Universidad Complutense de Madrid, 28040 Madrid, Spain. \\ ${ }^{b}$ Institute for Theoretical Physics, University of Heidelberg, 69120 Heidelberg, Germany \\ ${ }^{c}$ Institute of Theoretical Astrophysics, University of Oslo, 0315 Oslo, Norway
}

(Dated: November 3, 2009)

\begin{abstract}
It has been recently proposed that the presence of a temporal electromagnetic field on cosmological scales could explain the phase of accelerated expansion that the universe is currently undergoing. The field contributes as a cosmological constant and therefore, the homogeneous cosmology produced by such a model is exactly the same as that of $\Lambda$ CDM . However, unlike a cosmological constant term, electromagnetic fields can acquire perturbations which in principle could affect CMB anisotropies and structure formation. In this work, we study the evolution of inhomogeneous scalar perturbations in this model. We show that provided the initial electromagnetic fluctuations generated during inflation are small, the model is perfectly compatible with both CMB and large scale structure observations at the same level of accuracy as $\Lambda$ CDM.
\end{abstract}

PACS numbers: 98.80.-k, 95.36.+x, 14.70.Bh,04.62.+v

\section{INTRODUCTION}

Since the accelerated expansion of the universe was discovered more than a decade ago [1], a big effort has been made in trying to identify the culprit responsible for it. The simplest explanation is provided by the introduction of a cosmological constant term in the Einstein equations. In fact, such a solution is in excellent agreement with most of the cosmological observations to date and only requires the introduction of one extra parameter to describe the energy density associated to the cosmological constant. Hence, as a phenomenological model to describe the observable universe, it is very successful. However, the value of the cosmological constant inferred from the observations is extremely tiny as compared to the other scale present in the gravitational action and set by the Newton's constant, namely the Planck scale. This poses a problem from the theoretical point of view because a theory containing two dimensional constants which differ by so many orders of magnitude does not seem to be very natural. This problem is usually referred to as the naturalness problem or the cosmological constant problem, and it is related to the coincidence problem, that is, why dark energy and matter have similar density parameters today when their respective energy densities have evolved very differently throughout the expansion history of the universe. These two problems are the main reason why so many models to explain the current acceleration of the universe have been proposed. Among those models, one can generally find models in which the accelerated expansion is caused by either a new field (dark energy) or a modification of Einstein gravity. The popular quintessence models [2] with a cosmological scalar field playing the role of dark energy, and their generalizations with non-canonical kinetic terms, like the k-essence models [3] or with a coupling to curvature invariants [4] (see [5] for a review on dark energy models) they all belong to the first group. In the second group we have the very popular $f(R)$ theories [ [6] or the models with extra-dimensions [7]. Although all these models were motivated by the cosmological constant problem, the actual situation is that most of them fail in solving it because they rather shift it to some other parameter of the theory. Besides, they are usually plagued by either classical or quantum instabilities or present conflicts with local gravity tests [8].

Although the scalar fields have become the most popular candidates, vector fields have also been shown to be compelling candidates for dark energy [9, 10] and even solve the coincidence problem [11]. Generalisations to higher forms have also been considered recently [12]. Such models have been overlooked since they generically induce an anisotropy. This picture has changed in the last few years with the recent detections of some unexpected features in the CMB temperature anisotropies: A hemispherical asymmetry has been reported [13]; the angular correlation spectrum seems to be lacking power at the largest scales [14]; the alignment of the quadrupole and octupole (the so called Axis of Evil [15, 16]) could also be seem as an extra-ordinary and unlikely result of statistically isotropic perturbations, even without taking into account that these multipoles happen also to be aligned to some extent with the dipole and with the equinox. The significance of the anomalies has been debated extensively in the literature (see e.g. [17, 18, 19, 20, 21, 22, 23, 24]) with some reported effects more significant than others [25, 26, 27].

One model that, not only succeeds in addressing the cosmological constant problem, but also is free from both classical and quantum instabilities and has the same set of PPN parameters as General Relativity is the one recently proposed in [28]. In that model, the role of the cosmological constant is played by a temporal component of the 
electromagnetic field ${ }^{1}$ or, in other words, by the existence of an absolute cosmological electric potential [30]. That way, in addition of solving the aforementioned problems, we can establish the true nature of dark energy without resorting to unknown physics. In such a model, a slightly modified theory of the electromagnetic interaction is proposed and whose motivation comes from the quantization of the electromagnetic field in the covariant formalism. In that formalism, one modifies the electromagnetic action by introducing a gauge fixing term in the original action in exchange for either imposing the Lorenz condition $\partial_{\nu} A^{\nu}=0$ at the classical level or reducing the corresponding Hilbert space with the weak Lorenz condition $\partial^{\nu} A_{\nu}^{(+)}|\phi\rangle=0$ at the quantum level. However, when moving to an expanding universe, the Lorenz condition cannot be consistently imposed at all times and, therefore, this approach becomes inappropriate. For this reason, in the model proposed in [28], the electromagnetic interaction is quantized without imposing the Lorenz condition. The price to pay is that we need to introduce a new electromagnetic scalar mode in the theory so that the electromagnetic interaction would actually contain three physical degrees of freedom (see [31, 32] for other interesting consequences in theories with extra electromagnetic polarizations). This new scalar mode is however decoupled from the conserved currents and can only be excited by gravitational fields. Then, it could be generated during an inflationary phase in the early universe and the interesting feature of the model is that, once it becomes super-Hubble, it gives rise to a new contribution to the action which is like that of a cosmological constant. Surprisingly enough is the fact that the observed value of the cosmological constant is naturally achieved if inflation occurred at the electroweak scale.

Even though this model provides the same expansion history for the universe as a pure cosmological constant, its different nature makes possible to have a different evolution for the inhomogeneities so that it becomes necessary to perform an analysis of the perturbations to confront it to CMB and LSS data. In addition, as the observational data seem to be consistent with a cosmological term, it is then interesting that, by studying fluctuations, one in principle is able to infer the physical origin of the cosmological term. Previously the possibility of anisotropic fluctuations about a constant density term have been contemplated in the frameworks of viscous fluids and noncommutative geometry [10, 29]. Here the properties of such a cosmological term can be consistently derived from a fully covariant theory. The presence of fluctuations can be understood since the constancy of the cosmological term is just a property of a solution for a component of a vector field. Uncovering the evolution of the fluctuations is the aim of the present work, which is organized in the following way. In Section I we give a short description of the model. In Section II we write down the background equations and solve them. Besides, we show how the background evolution gives rise to the same expansion history as a cosmological constant. Section III is devoted to the perturbations for the electromagnetic dark energy model. In particular, we obtain the corresponding equations and perturbed expressions in the Newtonian gauge and solve them in some interesting cases. The analogous expressions for the synchronous gauge are given in an appendix. Finally, in section IV we use a modified version of the publicly available numerical code CAMB [35] to compute the evolution of the linear perturbations in the model. The results are summarized and discussed in the final Section VI.

\section{DESCRIPTION OF THE MODEL}

The proposed action describing the electromagnetic interaction including the gauge fixing term and current coupling is given by:

$$
S=\int d^{4} x \sqrt{g}\left[-\frac{1}{4} F_{\mu \nu} F^{\mu \nu}+\frac{\xi}{2}\left(\nabla_{\mu} A^{\mu}\right)^{2}+A_{\mu} J^{\mu}\right]
$$

with $F_{\mu \nu}=\partial_{\mu} A_{\nu}-\partial_{\nu} A_{\mu}$. Here, the gauge fixing term is considered as a fundamental piece of the action on equal footing to the Maxwell term, i.e. electromagnetism is considered as a gauge non-invariant theory which contains three physical degrees of freedom, namely the two usual transverse photons plus an extra scalar mode (despite the gauge non-invariance, the theory is perfectly consistent as shown in [28]). In fact, the gauge fixing term can be seen as the kinetic term of this new mode and so the parameter $\xi$ can be fixed to $1 / 3$ to have canonically normalized fields. While the general $U(1)$ transformations are no longer an exact symmetry of the theory, it still preserves a residual gauge symmetry given by $A_{\mu} \rightarrow A_{\mu}+\partial_{\mu} \theta$ provided $\square \theta=0$.

The modified Maxwell equations deduced from (1) read:

$$
\nabla_{\nu} F^{\mu \nu}+\xi \nabla^{\mu}\left(\nabla_{\nu} A^{\nu}\right)=J^{\mu}
$$

\footnotetext{
${ }^{1}$ We refer here to the vector $A_{\mu}$ as the electromagnetic field, instead of calling $A_{\mu}$ the vector potential and $F_{\mu \nu}$ the photon.
} 
If we now take the 4-divergence of these equations we obtain:

$$
\square\left(\nabla_{\nu} A^{\nu}\right)=0
$$

where we have used the fact that the electromagnetic current is covariantly conserved. The latter equation shows that $\nabla_{\nu} A^{\nu}$ behaves as a massless scalar field which is decoupled from the conserved electromagnetic currents, but it is non-conformally coupled to gravity so that it can be excited by gravitational fields. Moreover, due to the well-known fact that a massless scalar field gets frozen on super-Hubble scales for a FLRW universe, the four divergence of the electromagnetic field will be constant on scales larger than the Hubble radius, giving rise to a cosmological constantlike term in the action. On the other hand, for small scales we have that $\nabla_{\nu} A^{\nu} \sim a^{-1}$ so that its contribution to the Maxwell equations becomes negligible. Therefore, as long as the four divergence of the electromagnetic field is super-Hubble, it can play the role of a cosmological constant and, once it enters the horizon, it is rapidly suppressed and we recover the usual Maxwell equations with the Lorenz condition.

It is interesting to note that the divergence of the electromagnetic field can be seen, at the equations of motion level, as a conserved current acting as a source of the usual Maxwell field. To see this, we can write $-\xi \nabla^{\mu}\left(\nabla_{\nu} A^{\nu}\right) \equiv J_{\nabla \cdot A}^{\mu}$ which, according to (3), satisfies the conservation equation $\nabla_{\mu} J_{\nabla \cdot A}^{\mu}=0$ and we can express (2) as:

$$
\nabla_{\nu} F^{\mu \nu}=J_{T}^{\mu}
$$

with $J_{T}^{\mu}=J^{\mu}+J_{\nabla \cdot A}^{\mu}$ and $\nabla_{\mu} J_{T}^{\mu}=0$. Physically, it means that, while the new scalar mode can only be excited by means of gravitational fields, once it is produced it will generally be considered as a source of electromagnetic fields.

The energy-momentum tensor corresponding to the modified action (1) is the sum of two pieces:

$$
T_{\mu \nu}=T_{\mu \nu}^{M}+T_{\mu \nu}^{\xi}
$$

with

$$
T_{\mu \nu}^{M}=\frac{1}{4} g_{\mu \nu} F_{\alpha \beta} F^{\alpha \beta}-F_{\mu \alpha} F_{\nu}^{\alpha}
$$

the standard energy-momentum tensor corresponding to Maxwell theory and

$$
T_{\mu \nu}^{\xi}=\frac{\xi}{2}\left[g_{\mu \nu}\left[\left(\nabla_{\alpha} A^{\alpha}\right)^{2}+2 A^{\alpha} \nabla_{\alpha}\left(\nabla_{\beta} A^{\beta}\right)\right]-4 A_{(\mu} \nabla_{\nu)}\left(\nabla_{\alpha} A^{\alpha}\right)\right]
$$

the energy-momentum tensor corresponding to the gauge fixing term. Notice that, for a residual gauge mode of the form $A_{\mu}=\partial_{\mu} \theta$ with $\square \theta=0$, the energy-momentum tensor vanishes identically.

\section{BACKGROUND EQUATIONS}

We shall assume a homogeneous and isotropic background described by the FLRW metric which, in conformal time, is given by:

$$
d s^{2}=a(\tau)^{2}\left[d \tau^{2}-\delta_{i j} d x^{i} d x^{j}\right]
$$

In order to preserve the homogeneity we need the electromagnetic field to depend only on time whereas the large scale isotropy requires the absence of spatial components so that the background electromagnetic field is merely given by a temporal component which, in conformal time coordinates, we shall call $\mathcal{A}_{0}(\tau)^{2}$. Moreover, this homogeneous temporal component evolves according to the zero component of the equations (2), that yield:

$$
\mathcal{A}_{0}^{\prime \prime}+2\left(\mathcal{H}^{\prime}-2 \mathcal{H}^{2}\right) \mathcal{A}_{0}=0
$$

with ${ }^{\prime} \equiv \frac{d}{d \tau}$ and $\mathcal{H} \equiv \frac{a^{\prime}}{a}$ the Hubble expansion rate in conformal time. Notice that, since $F_{\mu \nu}=0$ for the assumed background configuration, the latter equation simply expresses that $d\left(\nabla_{\nu} A^{\nu}\right) / d \tau=0$ which, in turn implies that the four divergence of the electromagnetic field is constant irrespectively of the evolution of the scale factor.

\footnotetext{
${ }^{2}$ We shall denote by calligraphic letters the components of the vector field in conformal time coordinates
} 
If we use now the fact that $F_{\mu \nu}=0$ together with $\nabla_{\nu} A^{\nu}=$ const in the expression for the energy momentum tensor, we obtain that:

$$
T_{\mu \nu}=\frac{\xi}{2}\left(\nabla_{\mu} A^{\mu}\right)^{2} g_{\mu \nu}
$$

which is exactly the same as that of a cosmological constant of value $\Lambda=\frac{\xi}{2}\left(\nabla_{\nu} A^{\nu}\right)^{2}$. Notice that in order to have a positive cosmological constant we need $\xi>0$. The background evolution for this model thus mimics that of the standard $\Lambda$ CDM model whose cosmological constant value is determined by the four divergence of the electromagnetic field. The energy density of the electromagnetic field in this configuration is:

$$
\rho_{\mathcal{A}_{0}}=\frac{1}{6 a^{4}}\left(\mathcal{A}_{0}^{\prime}+2 \mathcal{H} \mathcal{A}_{0}\right)^{2}
$$

and the equation of state is simply $p_{A_{0}}=-\rho_{A_{0}}$. For the subsequent calculations, it will be convenient to introduce a redefinition of the vector field as $A_{0}=a^{2} \mathcal{A}_{0}$ so that expressions (9) and (11) look much simpler:

$$
\begin{array}{r}
\left(a^{-4} A_{0}^{\prime}\right)^{\prime}=0 \\
\rho_{A_{0}}=\frac{1}{6 a^{8}}\left(A_{0}^{\prime}\right)^{2}
\end{array}
$$

and we clearly see that the energy density is constant.

Finally, the fact that the background vector field does not contain spatial components is justified because on super-Hubble scales the spatial components grow more slowly than the temporal one. Let us see this in more detail. For a configuration with spatial components of the electromagnetic field in addition to the temporal one, i.e., with $\mathcal{A}_{\mu}=\left(\mathcal{A}_{0}(\tau), \overrightarrow{\mathcal{A}}(\tau)\right)$ and in a FLRW universe, the modified Maxwell equations read:

$$
\begin{aligned}
\mathcal{A}_{0}^{\prime \prime}+2\left(\mathcal{H}^{\prime}-2 \mathcal{H}^{2}\right) \mathcal{A}_{0} & =0 \\
\overrightarrow{\mathcal{A}}^{\prime \prime} & =0
\end{aligned}
$$

Thus, if we assume a power law expansion with $\mathcal{H}=p / \tau$, the solution of these equations is:

$$
\begin{aligned}
\mathcal{A}_{0} & =C_{01} \tau^{1+p}+C_{02} \tau^{p} \\
\overrightarrow{\mathcal{A}} & =\vec{C}_{1} \tau+\vec{C}_{2}
\end{aligned}
$$

and we see that the spatial components decay with respect to the temporal one in both the radiation $(p=1)$ and matter $(p=2)$ dominated eras. In addition, the energy density associated to the spatial part decays as $a^{-4}$ whereas that associated to the temporal component is constant, as we have already seen. Hence, it makes sense to assume the aforementioned isotropic background with vanishing spatial components given above.

\section{SCALAR PERTURBATION EQUATIONS}

In this section we shall derive the equations for the perturbations and solve them analytically for some simple cases. We only consider the case of scalar perturbations because we have checked that the vector perturbations evolve in the same way as in standard Maxwell theory and the tensor perturbations remain unaffected by the presence of the gauge fixing term as well. One might expect this because the gauge fixing term only affects the new scalar mode which, being scalar, can contribute to the scalar perturbations, but not to the pure transverse vector perturbations (i.e., the usual photons). We shall do the calculations of this section in the conformal Newtonian gauge, although we give the corresponding expressions for the synchronous gauge in the appendix.

In principle, the effect of the high-electric conductivity of the universe should be taken into account including the corresponding electromagnetic current in the r.h.s of Maxwell equations. The reason why we can neglect such effects is the following: the electromagnetic current should satisfy the conservation equation:

$$
\nabla_{\mu} J^{\mu}=0
$$

and also the condition of electric neutrality, i.e.

$$
u^{\mu} J_{\mu}=0
$$


with $u_{\mu}$ the four-velocity of the comoving observers. Let us expand also the current up to first order as:

$$
J_{\mu}=J_{\mu}^{0}+\delta J_{\mu}
$$

but $J_{\mu}^{0}=0$ for the homogeneous and isotropic electrically neutral background (this is the reason why we did not consider the current term in the background equations). If we now assume that the universe remains neutral at first order in the perturbations we have that $\delta J_{0}=0$ and, finally, from current conservation, we get: $\vec{\nabla} \cdot \overrightarrow{\delta J}=0$, i.e. the perturbed current is transverse. In other words, when computing scalar perturbations, we can ignore the effect of electric conductivity by assuming that the electric charge vanishes at first order as well.

In the considered gauge, the perturbed line element is given by:

$$
d s^{2}=a(\tau)^{2}\left[(1+2 \psi) d \tau^{2}-(1-2 \phi) \delta_{i j} d x^{i} d x^{j}\right]
$$

In the absence of any anisotropic stress sources we have $\phi=\psi$. In order to simplify the expressions we will use latter, we define the scalar perturbation of the vector field by introducing a convenient power of the scale factor as we did for the background field:

$$
\delta \mathcal{A}_{\mu}=a^{-2}\left(\delta \mathcal{A}_{0}, \nabla \delta \mathcal{A}\right)
$$

As usual, we shall introduce the Fourier components of the perturbations and solve the corresponding equations for them. Then, from (2) we can obtain the following equations for the Fourier modes of the vector field perturbations:

$$
\begin{aligned}
& \delta \mathcal{A}_{0 k}^{\prime \prime}-4 \mathcal{H} \delta \mathcal{A}_{0 k}^{\prime}-3 k^{2} \delta \mathcal{A}_{0 k}=-2 k^{2}\left(2 \delta \mathcal{A}_{k}^{\prime}-5 \mathcal{H} \delta \mathcal{A}_{k}\right)+\left[\left(\psi_{k}^{\prime \prime}-4 \mathcal{H} \psi_{k}^{\prime}+3 \phi_{k}^{\prime \prime}-12 \mathcal{H} \phi_{k}^{\prime}\right) A_{0}+3\left(\psi_{k}^{\prime}+\phi_{k}^{\prime}\right) A_{0}^{\prime}\right] \\
& \delta \mathcal{A}_{k}^{\prime \prime}-4 \mathcal{H} \delta \mathcal{A}_{k}^{\prime}+\left(4 \mathcal{H}^{2}-2 \mathcal{H}^{\prime}-\frac{1}{3} k^{2}\right) \delta \mathcal{A}_{k}=2\left(\frac{2}{3} \delta \mathcal{A}_{0 k}^{\prime}-\mathcal{H} \delta \mathcal{A}_{0 k}\right)-\left[\left(\frac{1}{3} \psi_{k}^{\prime}+\phi_{k}^{\prime}\right) A_{0}+\frac{2}{3} \psi_{k} A_{0}^{\prime}\right]
\end{aligned}
$$

In these equations we can see that the two perturbations of the vector field are coupled to each other and that the metric perturbations act as a source of them. Then, even in the case that the initial perturbations vanish the gravitational potentials will generate perturbations of the vector field that, eventually, may also be source of the metric perturbations.

On the other hand, the corresponding perturbed energy-momentum tensor components are given by:

$$
\begin{aligned}
\delta T_{0}^{0} & =\frac{1}{3 a^{8}}\left\{\left[-2 \psi_{k} A_{0}^{\prime}+\delta \mathcal{A}_{0 k}^{\prime}-\left(3 \phi_{k}^{\prime}+\psi_{k}^{\prime}\right) A_{0}\right] A_{0}^{\prime}+k^{2}\left[\left(3 \delta \mathcal{A}_{k}^{\prime}-6 \mathcal{H} \delta \mathcal{A}_{k}-3 \delta \mathcal{A}_{0 k}\right) A_{0}+\delta \mathcal{A}_{k} A_{0}^{\prime}\right]\right\} \\
\delta T^{i}{ }_{j} & =\frac{1}{3 a^{8}}\left\{\left[-2 \psi_{k} A_{0}^{\prime}+\delta \mathcal{A}_{0 k}^{\prime}-\left(3 \phi_{k}^{\prime}+\psi_{k}^{\prime}\right) A_{0}\right] A_{0}^{\prime}+k^{2}\left[\left(-3 \delta \mathcal{A}_{k}^{\prime}+6 \mathcal{H} \delta \mathcal{A}_{k}+3 \delta \mathcal{A}_{0 k}\right) A_{0}+\delta \mathcal{A}_{k} A_{0}^{\prime}\right]\right\} \delta^{i}{ }_{j} \\
\delta T^{0}{ }_{i} & =-\frac{i k_{i}}{3 a^{8}} A_{0}\left[-\left(3 \phi_{k}^{\prime}+\psi_{k}^{\prime}\right) A_{0}-2 \psi_{k} A_{0}^{\prime}+\delta \mathcal{A}_{0 k}^{\prime}+k^{2} \delta \mathcal{A}_{k}\right]
\end{aligned}
$$

It is interesting to note that this model has vanishing shear, i.e., $\delta T^{i}{ }_{j}=-\delta p_{k} \delta_{j}^{i}$. This is due to there being only one physical scalar mode present. Moreover, from expressions (24) one can find the following relation between the perturbed energy density and pressure of the field:

$$
\delta\left(\rho_{k}+p_{k}\right)=-\frac{2 A_{0} k^{2}}{a^{8}}\left(\delta \mathcal{A}_{0 k}-\delta \mathcal{A}_{k}^{\prime}+2 \mathcal{H} \delta \mathcal{A}_{k}\right)
$$

This relation is important because for a gauge mode satisfying $a^{-2} \delta \mathcal{A}_{\mu}=\partial_{\mu} \chi$ (remember here the definition of the perturbations given in (22) $)$ one has that $\delta \mathcal{A}_{0}=\delta \mathcal{A}^{\prime}+2 \mathcal{H} \delta \mathcal{A}$ and, as a consequence, $\delta\left(\rho_{k}+p_{k}\right)=0$.

In order to obtain some analytical results, in the following we shall consider that the metric perturbations are generated by some dominating fluid and study the evolution of the electromagnetic perturbations in such a scenario. In other words, we shall assume that the perturbations of the electromagnetic field will not affect the metric perturbations evolutions. This assumption is justified as long as the electromagnetic energy density is clearly subdominant as it happens in most of the universe evolution when the energy density associated to the electromagnetic field is many orders of magnitude below that of the dominant component. However, such a condition will eventually breakdown at low redshift when dark energy becomes dominant and the results obtained here lack validity, being necessary to resort to a numerical treatment. In the early universe when radiation represents the dominant contribution to the energy density of the universe and neglecting neutrinos shear (which implies that $\psi=\phi$ ) the metric perturbation evolve as [34]:

$$
\phi_{k}=\frac{C_{1 k}[\omega \tau \cos (\omega \tau)-\sin (\omega \tau)]+C_{2 k}[\omega \tau \sin (\omega \tau)+\cos (\omega \tau)]}{\tau^{3}}
$$


Vector field perturbation in the radiation era

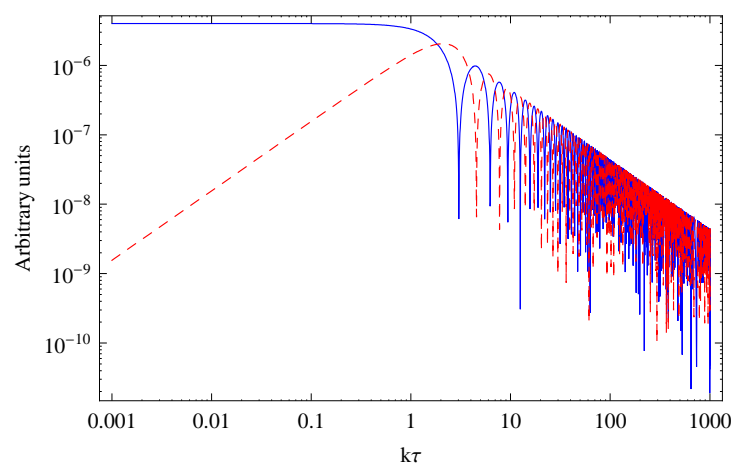

Vector field perturbation in the matter era

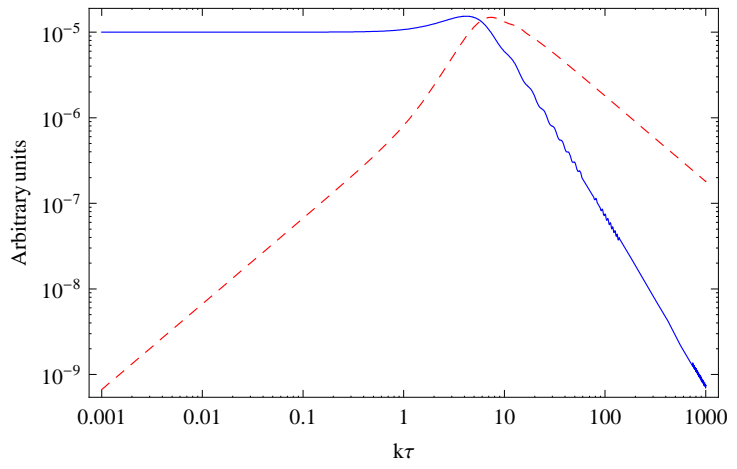

Energy-momentum perturbation in the radiation era

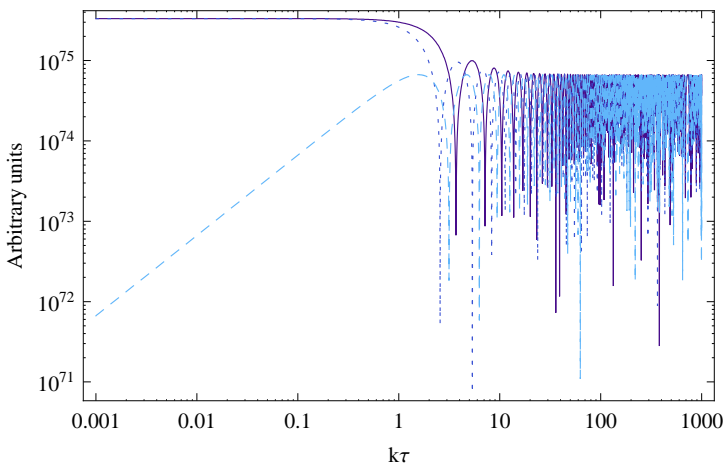

Energy-momentum perturbation in the matter era

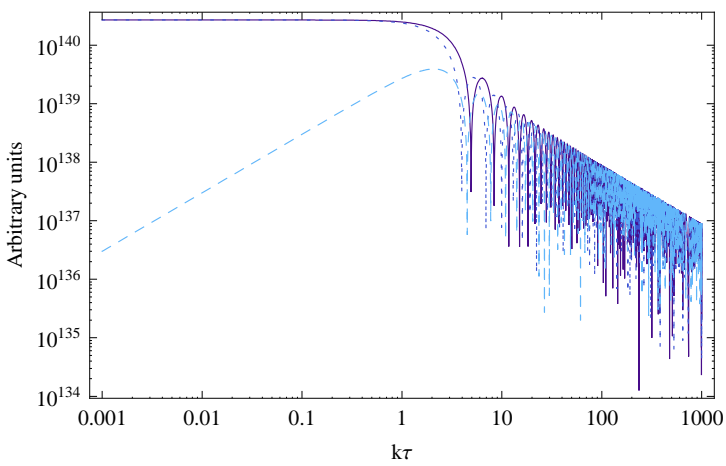

FIG. 1: In these figures we show the evolution of the vector field perturbations in the Newtonian gauge for radiation (upper panel) and matter (lower panel) dominated universes. In the left panels we show the evolution of the vector field perturbations $\delta \mathcal{A}_{0} / A_{0}$ (solid-blue) and $k \delta \mathcal{A} / A_{0}$ (dashed-red). The right panels show the perturbed energy density $\delta T_{0}^{0}$ (solid), pressure $-\frac{1}{3} \delta T_{i}^{i}$ (dotted) and momentum $-\frac{1}{i k^{2}} k_{i} \delta T_{i}^{0}$ (dashed).

with $\omega=k / \sqrt{3}$. On the other hand, in the matter dominated universe, the gravitational potential becomes constant in time, i.e., $\phi_{k}=$ const. Then, we can solve the equations (23) in the presence of the gravitational perturbations produced by a radiation or matter fluid and obtain the evolution of the vector field perturbations in those epochs. The results are shown in Fig. 1. We can see that, on super-Hubble scales, the perturbation $\delta \mathcal{A}_{0}$ evolves in the same way as the background vector field so that $\delta \mathcal{A}_{0} / A_{0}=$ const as one would expect. This also implies that the perturbed energy density is constant on large-scales as we can see in the figure. Moreover, this feature does not depend on the dominating fluid, i.e., it happens for both the radiation and matter eras. On small scales, the perturbed energy density scales with constant amplitude when the universe is dominated by radiation whereas the amplitude decays as $1 / \tau$ in the matter era. Notice that this behavior on small scales is a common feature for all the perturbed components of the energy-momentum tensor, i.e., the energy, the pressure and the scalar component of the momentum.

We shall obtain the explicit evolution for the electromagnetic perturbations during the matter-dominated era, when most of the cosmologically relevant scales reenter the horizon. In that epoch, the gravitational potential is constant as we said above so that $\phi_{k}=\psi_{k}=\phi_{0}$ and the Hubble parameter satisfies $\mathcal{H}^{\prime}=-\frac{1}{2} \mathcal{H}^{2}$. With these conditions, we can obtain the following expression for $\delta \mathcal{A}$ in terms of $\delta \mathcal{A}_{0}$ and $\phi_{0}$ :

$$
\delta \mathcal{A}_{k}=-\frac{3}{4 k^{2}}\left[\delta \mathcal{A}_{0 k}^{\prime \prime \prime}-\frac{11}{2} \mathcal{H} \delta \mathcal{A}_{0 k}^{\prime \prime}+\left(\frac{7}{3} k^{2}-8 \mathcal{H}^{2}\right) \delta \mathcal{A}_{0 k}^{\prime}-\frac{7}{2} \mathcal{H} k^{2} \delta \mathcal{A}_{0 k}-\frac{8}{3} A_{0}^{\prime} \phi_{0}\right]
$$

This relation allows to find the following fourth-order differential equation for $\delta \mathcal{A}_{0 k}$ :

$$
\delta \mathcal{A}_{0 k}^{\mathrm{iv}}-8 \mathcal{H} \delta \mathcal{A}_{0 k}^{\prime \prime \prime}+2\left(k^{2}-\frac{49}{4} \mathcal{H}^{2}\right) \delta \mathcal{A}_{0 k}^{\prime \prime}-8 \mathcal{H}^{2}\left(k+\frac{7}{2} \mathcal{H}\right) \delta \mathcal{A}_{0 k}^{\prime}+k^{2}\left(k^{2}+\frac{21}{2} \mathcal{H}^{2}\right) \delta \mathcal{A}_{0 k}=4 k^{2} \mathcal{H} A_{0}^{\prime} \phi_{0}
$$

This equation together with the relation (27) determine the evolution of the electromagnetic perturbations in a matter dominated universe. It is convenient to remind here the assumptions under which such equations remain valid. In 
order to obtain those equations we have assumed that the metric perturbations act as an external source for the electromagnetic perturbations and that this external source is uniquely determined by the matter fluid. This means that the contribution of the electromagnetic field to the perturbed Einstein equations are negligible with respect to that of the matter component, which is a good approximation as long as the electromagnetic field energy density is well below the matter energy density. However, this condition eventually breakdowns because the electromagnetic field becomes dominant and it contributes in a non-negligible way to the Einstein equations so that the metric perturbations becomes affected by the electromagnetic field perturbations and the full system of coupled equations must be solved.

In order to solve eq. (28) we shall take advantage of the residual gauge symmetry of the theory, namely, $A_{\mu} \rightarrow$ $A_{\mu}+\partial_{\mu} \theta$ with $\square \theta=0$. From this symmetry, we know that $\delta \mathcal{A}_{0}=a^{2} \theta^{\prime}$ will be solution of (28) so that we can factorize it as $\mathcal{F}\left[\mathcal{G}\left(\delta \mathcal{A}_{0}\right)\right]=4 k^{2} \mathcal{H} A_{0}^{\prime} \phi_{0}$, where $\mathcal{F}$ is a second order differential operator and $\mathcal{G}$ is the operator determining the evolution of $a^{2} \theta^{\prime}$ which can be deduced from the equation satisfied for $\theta$ and turns out to be $\mathcal{G}=\frac{d^{2}}{d \tau^{2}}-2 \mathcal{H} \frac{d}{d \tau}+k^{2}$. Therefore, the equation (28) can be expressed as:

$$
\left[\frac{d^{2}}{d \tau^{2}}-6 \mathcal{H} \frac{d}{d \tau}+\left(k^{2}+\frac{21}{2} \mathcal{H}^{2}\right)\right]\left[\frac{d^{2}}{d \tau^{2}}-2 \mathcal{H} \frac{d}{d \tau}+k^{2}\right] \delta \mathcal{A}_{0 k}=4 k^{2} \mathcal{H} A_{0}^{\prime} \phi_{0}
$$

Thus, the solution for $\delta \mathcal{A}_{0}$ will be determined by the equation $\mathcal{G}\left(\delta \mathcal{A}_{0}\right)=S$ with $S$ the solution of the equation $\mathcal{F}(S)=4 k^{2} \mathcal{H} A_{0}^{\prime} \phi_{0}$. Since $\mathcal{H}=\frac{2}{\tau}$ in the matter era, we can obtain the explicit form of the kernel of $\mathcal{F}$ (solutions of the homogeneous equation) and is given by:

$$
S_{h o m}(\tau)=\tau^{6}\left(C_{S 1} e^{-i k \tau}+C_{S 2} e^{i k \tau}\right)
$$

whereas a particular one can be obtained by:

$$
S_{\text {part }}=S_{1} \int \frac{S_{2}}{\operatorname{det} W_{S}} 4 k^{2} \mathcal{H} A_{0}^{\prime} \phi_{0} d \tau-S_{2} \int \frac{S_{1}}{\operatorname{det} W_{S}} 4 k^{2} \mathcal{H} A_{0}^{\prime} \phi_{0} d \tau
$$

where $S_{1}$ and $S_{2}$ are the two independent solutions given above and $\operatorname{det} W_{S}=S_{1}^{\prime} S_{2}-S_{2}^{\prime} S_{1}=2 i k \tau^{12} C_{S 1} C_{S 2}$ is the determinant of the Wronskian. Following the same procedure, we obtain the kernel of $\mathcal{G}$

$$
\delta \mathcal{A}_{0}^{h o m}(\tau)=C_{1}\left(k^{2} \tau^{2}-3 i k \tau-3\right) e^{-i k \tau}+C_{2}\left(k^{2} \tau^{2}+3 i k \tau-3\right) e^{i k \tau}
$$

whereas the particular solution is

$$
\delta \mathcal{A}_{0}^{\text {part }}=\delta \mathcal{A}_{0, C_{1}} \int \frac{\delta \mathcal{A}_{0, C_{2}}}{\operatorname{det} W_{C}} S(\tau) d \tau-\delta \mathcal{A}_{0, C_{2}} \int \frac{\delta \mathcal{A}_{0, C_{1}}}{\operatorname{det} W_{C}} S(\tau) d \tau
$$

with $\delta \mathcal{A}_{0, C_{i}}(i=1,2)$ denoting the piece of the homogeneous solution (32) proportional to $C_{i}(i=1,2)$ and det $W_{C}=$ $2 i k^{5} \tau^{4} C_{1} C_{2}$. The homogeneous solution corresponds to the pure gauge degree of freedom and it does not contribute to the perturbed energy density, whereas the particular solution gives rise to the oscillating behavior with an amplitude decaying as $\tau^{-1}$ for small scales shown in Fig. 11.

We would like to remark that the procedure followed in order to solve the equations for a matter dominated universe is completely general and can be applied in any other situation where the metric perturbations is originated by some other component.

\section{EVOLUTION OF THE PERTURBATIONS}

In this section we shall present the results that we obtain from a modified version of the publicly available CAMB code [35] to compute the CMB power spectrum when electromagnetic perturbations are taken into account. Since the background of the electromagnetic model is the same as that of $\Lambda$ CDM, we do not need to modify the background equations of the code, although we do have to add the evolution equation for $A_{0}$. We use the first order equation (13) so that we can relate the background electromagnetic field directly to the present value of its density parameter. The initial condition for this equation is unimportant for the background evolution, although it becomes relevant for the perturbations and it is set by assuming a power law behavior for $A_{0}$. For the perturbation equations, we add the two evolution equations for the electromagnetic perturbations given by (50) and modify the corresponding terms involving dark energy perturbations. With these modifications, the code is ready to compute the evolution of the perturbations in the cosmology corresponding to the electromagnetic model and, thus, obtain the CMB power spectrum as well as the matter power spectrum. 
Before proceeding to show the obtained results, we shall discuss what the initial conditions for the electromagnetic field perturbations should be. A natural origin for the presence of the new mode of the electromagnetic field on cosmological scales has been proved to be the quantum fluctuations of such a mode during an inflationary epoch [28]. In such a scenario, only the new scalar mode can be excited because of the conformal invariance of the usual transverse modes. The "homogeneous part" of this scalar mode, defined as the sum of all the modes which remain super-Hubble today, gives rise to the effective cosmological constant whereas the modes which have already reentered into the horizon constitute the origin of the electromagnetic perturbations discussed in the present work. In other words, we can split the primordial quantum fluctuations of the scalar mode generated during inflation in a homogeneous part comprising all the modes with $k<k_{0}$ (being $k_{0}$ the scale that is entering into the horizon today, i.e., the present Hubble radius) and an inhomogeneous part formed by those modes with $k>k_{0}$. Notice that such a split can be performed because the primordial power spectrum for the scalar mode is red-tilted, as shown in [28], so that the homogeneous part is large as compared to the inhomogeneous one and this enable us to treat the latter as a perturbation. For the mentioned scalar mode, one can see that the longitudinal component decays with respect to the temporal component on super-Hubble scales for a de-Sitter inflationary epoch (in an analogous manner to that shown for the background evolution) so that, at the end of inflation, the amplitude of the longitudinal component would be expected to be much smaller than the temporal one. Moreover, we have already shown that the longitudinal components also decay with respect to the temporal one in the radiation dominated epoch so that the super-Hubble modes would be expected to be strongly suppressed at the time when the initial conditions are given, which justifies to set the initial condition for $\delta \mathcal{A}_{k}$ to zero. On the other hand, the power spectrum of the quantum fluctuations generated during a de Sitter inflation for the temporal component happens to be scale-invariant ${ }^{3}$ so that we can set the initial condition for $\delta \mathcal{A}_{0 k}$ as $\delta \mathcal{A}_{0 k}\left(\tau_{i n i}\right)=A k^{-3 / 2}$ with $A$ a constant depending on the details of the inflationary epoch such as the initial amplitude of the power spectrum after inflation or the duration of inflation. For our purposes in this work, this constant $A$ will play the role of a free parameter to be constrained by comparing the CMB power spectrum produced by the model to the WMAP data. In the same way as for the background vector field, we shall give the initial condition for the derivative of $\delta \mathcal{A}_{0 k}$ by assuming a power-law behavior. This is justified because we know that this is the type of evolution for the perturbations on super-Hubble scales, where the initial conditions are given.

In Fig. 2 and Fig. 3 we show the results obtained from the modified version of the CAMB code. The modifications in both the $\mathrm{CMB}$ and matter power spectrum is originated thanks to the fact that, unlike in the cosmological constant case, the electromagnetic dark energy model produces fluctuations that might modify the evolution of the gravitational potential. In that figure, we can see that the small scales behavior is unaffected with respect to the standard $\Lambda \mathrm{CDM}$ case. The reason for this is that the electromagnetic perturbations decay very rapidly once they enter into the horizon as we have shown in the previous section so that only those electromagnetic modes whose scales have become sub-Hubble very recently (corresponding to the low multipoles part of the spectrum) can contribute in a non-negligible way to the metric perturbation evolution through Einstein equations. Moreover, since dark energy density is negligible during decoupling, the contribution of electromagnetic perturbations to the ordinary Sachs-Wolfe effect is also negligible. The main effect will appear in the late-time Integrated Sachs-Wolfe (ISW) effect as due to the evolution of the metric perturbation. The analytical estimate of such an effect is difficult to obtain since it requires to know the time evolution of the metric perturbation when the electromagnetic perturbations contribute in a non-negligible way to the Einstein equations, so that the approximated solutions obtained in the previous section are no longer valid. However, we can easily understand how the ISW effect will be modified by noticing that those modes that have become sub-Hubble very recently can still have an appreciable amplitude and, therefore, modify the late-time evolution of the gravitational potentials, which gives rise to a modification of the ISW, but only for low redshift so that the early ISW remains unaffected. Moreover, if the corresponding mode crosses the horizon with a too large amplitude, the modification in the gravitational potentials evolutions might be excessively large and, thus, conflict with observations. This is the reason why on large scales we obtain some distinctive signatures for large enough values of the primordial amplitude $A$. Notice that such signatures are more apparent in the matter power spectrum. Instead of giving the constraints in terms of the parameter $A$ we shall give the results in terms of the more physical quantity $\delta_{A}$ defined as:

$$
\delta_{A} \equiv \frac{\mathcal{P}_{k}^{1 / 2}}{\rho_{\mathcal{A}_{0}}}
$$

\footnotetext{
${ }^{3}$ As we have already commented, in a more realistic quasi-de-Sitter inflationary epoch, the power spectrum of the temporal component becomes slightly red-tilted with a spectral index $n_{A_{0}}=\mathcal{O}(\varepsilon)$ with $\varepsilon$ the slow-roll parameter [28]. However, we shall neglect this small spectral index in order to set the initial conditions, being its effect expected to be small.
} 
with $\mathcal{P}_{k}=\frac{k^{3}}{2 \pi^{2}}\left|\delta \rho_{k}\right|^{2}$ the power spectrum of the electromagnetic energy density perturbations. Hence, the magnitude $\delta_{A}$ gives the amplitude of the energy density fluctuations of the electromagnetic field at a given scale $k$ relative to the homogeneous contribution. Since $\delta \rho_{k}$ and $\rho_{\mathcal{A}_{0}}$ evolve in the same way on super-Hubble scales, the quantity $\delta_{A}$ does not depend on time as long as the corresponding mode remains super-Hubble. Notice also that $\delta \rho_{k}$ contains two types of contributions, namely: one proportional to the metric perturbation and other proportional to the electromagnetic perturbation. Thus, in the case when the component proportional to the electromagnetic perturbation becomes dominant, the quantity $\delta_{A}$ becomes scale-invariant on super-Hubble scales because $\delta \rho_{k} \propto \delta \mathcal{A}_{0 k}$ and $\delta \mathcal{A}_{0 k}$ is proportional to $k^{-3 / 2}$ due to the flatness of its primordial power spectrum. However, if the metric perturbation contribution is dominant $\delta_{A}$ will depend on the wave-number of the considered mode. In any case, the upper bound that we shall obtain for $\delta_{A}$ will show how large the primordial electromagnetic perturbations are allowed to be in order to be compatible with CMB measurements. In particular, we obtain that $\delta_{A}$ must satisfy the constraint $\delta_{A} \lesssim 10^{-7}$ in order not to be in conflict with the CMB quadrupole. In fact, the overall effect on the CMB power spectrum would be that the higher value of $\delta_{A}$ is, the more tilted the lower multipoles part of the spectrum becomes. In fact, since the $\delta \rho_{k}$ modes decay rapidly once they reenter into the horizon (as we already commented above) the only important effect appears for the quadrupole and, as a consequence, the bound on $\delta_{A}$ is actually a bound on such a quantity at the quadrupole scale.

On the other hand, the upper bound obtained for $\delta_{A}$ can be linked to a variation of the Hubble parameter in a quasi-de-Sitter inflationary epoch where the the Hubble parameter is not exactly constant but varies slightly. To show such a link, we have to remind that the background energy density of the electromagnetic field is given by all the modes whose scales are larger than the Hubble radius today, whereas the perturbations correspond to modes whose scales are smaller than the present Hubble radius, as commented before. In other words, the background is given by the modes that remain super-Hubble at the present epoch and the perturbations correspond to those modes which have already entered into the horizon. In [28] we showed that the amplitude of the electromagnetic field fluctuations (for the temporal component) at a scale $k$ is given by $H_{k}$, with $H_{k}$ the Hubble parameter at the time when the scale $k$ exits from the horizon. Now, let us notice that

$$
\delta_{A} \sim \frac{H_{k}^{2} A_{0} \delta \mathcal{A}_{0 k}}{H_{I}^{2} A_{0}^{2}} \sim\left(\frac{H_{k}}{H_{I}}\right)^{3}
$$

where $H_{I}$ is the Hubble parameter at the beginning of inflation. Then, the constraint $\delta_{A} \lesssim 10^{-7}$ implies that the Hubble parameter must have reduced (at least) by a factor $\sim 200$ since the beginning of inflation until the time when the scale of the present Hubble radius (quadrupole scale) left the horizon, i.e., $H_{k_{0}} \lesssim H_{I} / 200$. Since the Hubble parameter is proportional to the square of the inflation scale we also obtain that the scale of inflation must reduce in a factor $\sim 15$. Notice also that this fact requires a red-tilted spectrum for inflation, i.e., the Hubble parameter must decay throughout the inflationary epoch. Moreover, since $\delta \mathcal{A}_{0 k} \sim H_{k}$ (as we have already said) we have that the fluctuations of the temporal component must satisfy:

$$
\frac{\delta \mathcal{A}_{0 k}}{A_{0}} \lesssim 5 \times 10^{-3}
$$

Then, we conclude that the electromagnetic dark energy model is compatible with current CMB and LSS unless very large electromagnetic initial perturbations are generated during inflation.

\section{DISCUSSION}

In the quantization of the electromagnetic field, either in the covariant or in the path-integral formalism, one has to introduce a gauge-fixing term in order to eliminate unphysical modes. The form of the gauge fixing term is unique in the sense that it is the only one that does not require the introduction of additional scales in the action and leads to linear equations of motion, although one may even consider nonlinear terms, in which case Faddeev-Popov ghosts appear also in flat space-time quantum electrodynamics. It has been proposed that the familiar, linear gauge-fixing term might have physical consequences in curved spacetime. One then promotes the gauge-fixing term into physical term which is included in the fundamental theory and not introduced during the quantization procedure as usually. In flat space, the usual predictions of electromagnetism are retained. However, a gravitational field may excite a scalar degree of freedom of the electromagnetic field, which now is not a gauge mode but has physical consequences. A remarkable result of this is that in the homogeneous and isotropic background the temporal component of the vector $A_{\mu}$ assumes an evolution that conspires with the cosmological expansion to contribute as an effective cosmological constant. This constant is of the order of magnitude required by the present cosmological data given that the field was generated during inflation occurring at the electroweak scale. 

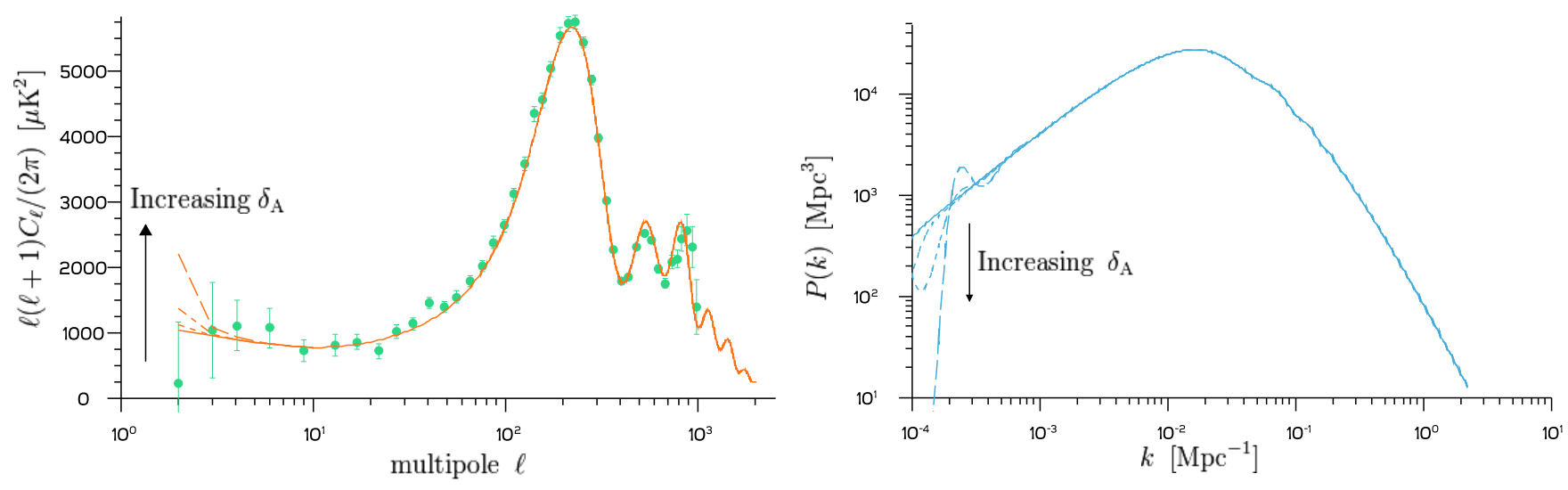

FIG. 2: In this figure we show the CMB power spectrum (left panel) and the matter power spectrum (right panel) for both the electromagnetic dark energy model (dotted lines) and the standard $\Lambda$ CDM model (solid lines). We have plotted several cases with increasing values of the initial amplitude for the electromagnetic perturbations and we see that the only modifications appear for large scales. In particular, the CMB quadrupole becomes excessive large, being incompatible with WMAP data (green dots) for $\delta_{A}>10^{-7}$ and the small $k$ region of the matter power spectrum becomes very different from that of $\Lambda$ CDM.
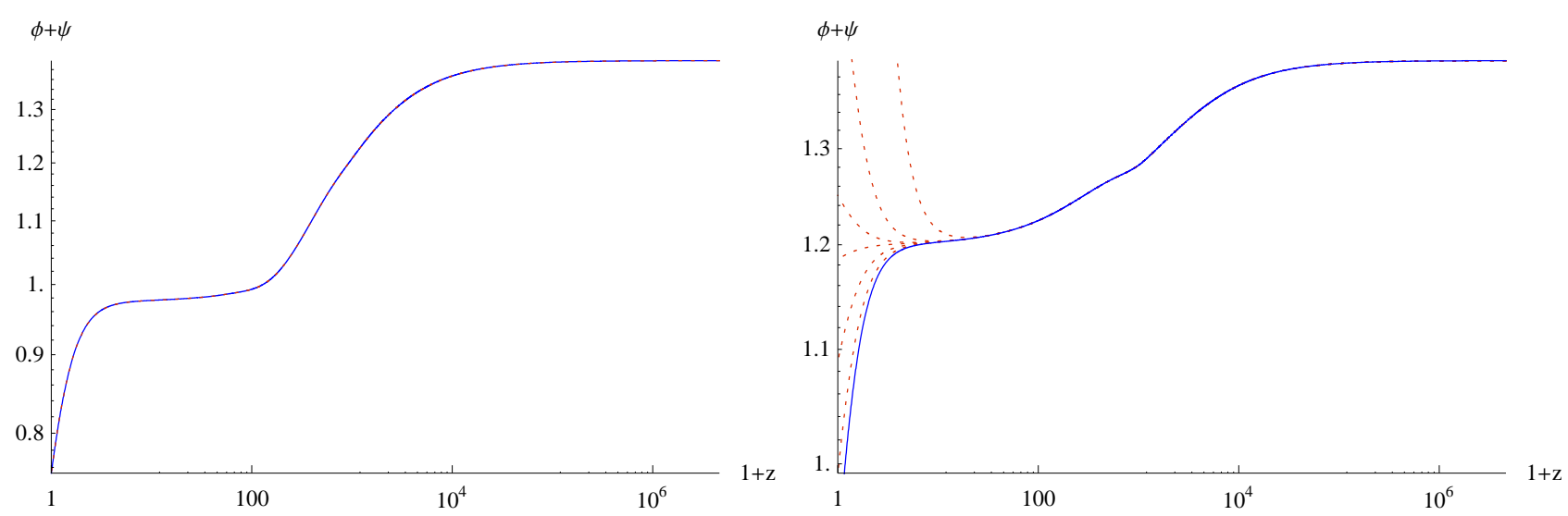

FIG. 3: In this figure we show the evolution of the gravitational potentials for small $\left(k=5.8 \times 10^{-3} \mathrm{Mpc}^{-1}\right)$ and large $\left(k=1.1 \times 10^{-5} \mathrm{Mpc}^{-1}\right)$ scales in the left and right panels respectively. We also show the evolution in a $\Lambda$ CDM model (solid blue lines) for comparison. As commented in the main text, the evolution for the small scales is exactly the same as in the case of a cosmological constant. In the large scales case we see how the evolution can be very different for large enough initial amplitudes of the electromagnetic perturbations. We plot the electromagnetic model in dotted (red) lines and we see that the larger the electromagnetic perturbations are, the more distinctive the evolution of the gravitational potential is.

Such a term, being not covariantly constant but having internal dynamics, implies there will be fluctuations. We analysed these fluctuations both analytically and numerically. At superhorizon scales, the perturbations tend to freeze to constant value, while after entering horizon, they decay exhibiting oscillations. As the cosmological constant begins to contribute more significantly to the energy density, the fluctuations of the field begin to affect the gravitational potentials. Since this happens at recent times, the effects to the matter power spectrum is confined to large scales, and to the CMB at small multipoles. However, depending on the initial amplitude of the perturbations, which in turn depends on the details of inflation, these effects might be detectable. In terms of the parameter (34) we find $\delta_{A} \lesssim 10^{-7}$. This implies that the amplitude of fluctuations generated for the electromagnetic field is $\lesssim 5 \times 10^{-3}$. Moreover, this fact predicts a reduction of the scale of inflation in a factor (at least) $\sim 15$ since the beginning of inflation until the scale of the quadrupole crossed the horizon. Since the mode $\nabla_{\mu} A^{\mu}$ behaves as a free test scalar field, see Eq.(3), we expect that it acquires the spectrum whose amplitude is given by the Hubble parameter just like that of the inflaton field (with just a slightly different slope due to negligible backreaction).

This shows that the model can be perfectly viable, but even more interestingly, for some specific inflationary scenarios there could be observable signatures from the model. Initial fluctuations of the order $\gtrsim 10^{-3}$ can already be ruled out. In addition, if a coupling existed between the inflaton and the electromagnetic field, or if the temporal 
component of the latter contributed non-negligibly to the inflationary expansion, one would expect their spectra to be correlated, which could yet lead to a new kind of signatures.

Acknowledgements: This work has been supported by Ministerio de Ciencia e Innovación (Spain) project numbers FIS 2008-01323 and FPA 2008-00592, UCM-Santander PR34/07-15875. J.B. wishes to thank the Institute for Theoretical Physics of the University of Heidelberg for their hospitality.

[1] A.G. Riess et al., Astron. J.116: 1009-1038 (1998); S. Perlmutter et al., Astrophys. J. 517: 565-586 (1999).

[2] C. Wetterich, Nucl. Phys. B302: 668 (1988); R.R. Caldwell, R. Dave and P.J. Steinhardt, Phys. Rev. Lett. 80: 1582 (1998)

[3] C. Armendariz-Picon, T. Damour and V. Mukhanov, Phys. Lett. B458: 209 (1999)

[4] T. Koivisto and D. F. Mota, Phys. Rev. D 75, 023518 (2007); Phys. Lett. B 644, 104 (2007).

[5] E. J. Copeland, M. Sami and S. Tsujikawa, Int. J. Mod. Phys. D15: 1753-1936 (2006).

[6] S.M. Carroll, V. Duvvuri, M. Trodden, M.S. Turner, Phys. Rev. D70: 043528, (2004).

[7] G. Dvali, G. Gabadadze and M. Porrati, Phys. Lett. B485: 208 (2000)

[8] R. Durrer and R. Maartens, arXiv:0811.4132 [astro-ph].

[9] V.V. Kiselev, Class. Quant. Grav. 21: 3323 (2004). C. Armendariz-Picon, JCAP 0407: 007 (2004); C.G. Boehmer and T. Harko, Eur. Phys. J. C50: 423, (2007); M. Novello, et al. Phys. Rev. D69: 127301, (2004); T. Koivisto and D.F. Mota, Astrophys. J. 679: 1 (2008). H.S. Zhao, Astrophys. J., 671, L1-L4 (2007); K. Bamba, S. Nojiri and S. D. Odintsov,Phys. Rev. D77, 123532 (2008); T. S. Koivisto and D. F. Mota, JCAP 0808, 021 (2008); J. Beltrán Jiménez and A.L. Maroto, arXiv:0905.1245 [astro-ph.CO].

[10] T. Koivisto and D. F. Mota, JCAP 0806, 018 (2008); D. F. Mota, J. R. Kristiansen, T. Koivisto and N. E. Groeneboom, Mon. Not. Roy. Astron. Soc. 382, 793 (2007): T. Koivisto and D. F. Mota, Phys. Rev. D 73, 083502 (2006): B. Li et al. Phys. Rev. D 77, 024032 (2008).

[11] J. Beltrán Jiménez and A.L. Maroto, Phys. Rev. D78: 063005 (2008); J. Beltrán Jiménez and A.L. Maroto, ePrint: arXiv:0807.2528 [astro-ph]; J. Beltrán Jiménez, R. Lazkoz and A.L. Maroto, Phys. Rev. D80: 023004 (2009), arXiv:0904.0433 [astro-ph.CO].

[12] C. Germani and A. Kehagias, JCAP 0903, 028 (2009); T. S. Koivisto, D. F. Mota and C. Pitrou, e-PrintarXiv:0903.4158 [astro-ph.CO].

[13] H. K. Eriksen, F. K. Hansen, A. J. Banday, K. M. Gorski, and P. B. Lilje, Asymmetries in the CMB anisotropy field, Astrophys. J. 605 (2004) 14-20.

[14] WMAP Collaboration, G. Hinshaw et. al., Astrophys. J. Suppl. 170 (2007) 288.

[15] K. Land and J. Magueijo, The axis of evil, Phys. Rev. Lett. 95 (2005) 071301.

[16] A. Rakic and D. J. Schwarz, Phys. Rev. D75 (2007) 103002.

[17] G. Efstathiou, Mon. Not. Roy. Astron. Soc. 348 (2004) 885.

[18] A. de Oliveira-Costa, M. Tegmark, M. Zaldarriaga, and A. Hamilton, Phys. Rev. D69 (2004) 063516.

[19] J. Magueijo and R. D. Sorkin, Mon. Not. Roy. Astron. Soc. Lett. 377 (2007) L39-L43.

[20] P. Bielewicz, K. M. Gorski, and A. J. Banday, Mon. Not. Roy. Astron. Soc. 355 (2004) 1283.

[21] S. L. Bridle, A. M. Lewis, J. Weller, and G. Efstathiou, Mon. Not. Roy. Astron. Soc. 342 (2003) L72.

[22] D. J. Schwarz, G. D. Starkman, D. Huterer, and C. J. Copi, Phys. Rev. Lett. 93 (2004) 221301.

[23] A. Slosar, U. Seljak, and A. Makarov, Phys. Rev. D69 (2004) 123003.

[24] S. Prunet, J.-P. Uzan, F. Bernardeau, and T. Brunier, Phys. Rev. D71 (2005) 083508.

[25] J. Hoftuft et. al., arXiv:0903.1229 [astro-ph.CO].

[26] F. K. Hansen, A. J. Banday, K. M. Gorski, H. K. Eriksen, and P. B. Lilje, arXiv:0812.3795 [astro-ph].

[27] N. E. Groeneboom and H. K. Eriksen, Astrophys. J. 690 (2009) 1807-1819.

[28] J. Beltrán Jiménez and A.L. Maroto, JCAP 0903: 016 (2009), e-Print: arXiv:0811.0566 [astro-ph]; J. Beltrán Jiménez and A.L. Maroto, e-Print: arXiv:0903.4672 [astro-ph.CO]; J. Beltrán Jiménez and A.L. Maroto, JCAP 0902:025, (2009), e-Print: arXiv:0811.0784 [astro-ph]; J. Beltrán Jiménez and A.L. Maroto, e-Print: arXiv:0812.1970 [astro-ph].

[29] D. C. Rodrigues, Phys. Rev. D 77 (2008) 023534 arXiv:0708.1168 [astro-ph]].

[30] J. Beltrán Jiménez and A.L. Maroto, arXiv:0905.2589 [astro-ph.CO].

[31] M. S. Turner and L. M. Widrow, Inflation Produced, Large Scale Magnetic Fields, Phys. Rev. D37 (1988) 2743.

[32] O. Bertolami and D. F. Mota, Primordial Magnetic Fields via Spontaneous Breaking of Lorentz Invariance, Phys. Lett. B455 (1998) 96.

[33] C.P. Ma and E. Bertschinger, Astrophys. J. 455: 7-25 (1995).

[34] V.F. Mukhanov, H.A. Feldman and R.H. Brandenberger, Phys. Rept. 215: 203-333 (1992).

[35] A. Lewis, A. Challinor and A. Lasenby, Astrophys. J. 538 (2000) 473 arXiv:astro-ph/9911177.

[36] J. M. Stewart and M. Walker, Proc. Roy. Soc. Lond. A 341 (1974) 49. 


\section{APPENDIX: PERTURBATION EQUATIONS IN THE SYNCHRONOUS GAUGE}

In this appendix we shall derive the equations of the perturbations and all the other expressions in the synchronous gauge, for which the perturbed line element is:

$$
d s^{2}=a(\tau)^{2}\left[d \tau^{2}-\left(\delta_{i j}+h_{i j}\right) d x^{i} d x^{j}\right]
$$

The scalar modes of the perturbation are usually expressed in terms of the scalar functions $h$ and $\eta$ defined by means of [33]:

$$
h_{i j}^{(s)}=\int d^{3} k\left[\frac{k_{i} k_{j}}{k^{2}} h_{k}(\tau)+\left(\frac{k_{i} k_{j}}{k^{2}}-\frac{1}{3} \delta_{i j}\right) 6 \eta_{k}(\tau)\right] e^{i \vec{k} \cdot \vec{x}}
$$

To go from one gauge to another we perform a coordinate transformation given by:

$$
\hat{x}^{\mu}=x^{\mu}+\zeta^{\mu}
$$

As we are considering only scalar perturbations we can set $\zeta^{\mu}=(\alpha, \nabla \beta)$. The relation between the scalar metric perturbations in both gauges is as follows [33]:

$$
\begin{aligned}
\psi_{k} & =\frac{1}{2 k^{2}}\left[h_{k}^{\prime \prime}+6 \eta_{k}^{\prime \prime}+\mathcal{H}\left(h_{k}^{\prime}+6 \eta_{k}^{\prime}\right)\right] \\
\phi_{k} & =\eta_{k}-\frac{1}{2 k^{2}} \mathcal{H}\left(h_{k}^{\prime}+6 \eta_{k}^{\prime}\right)
\end{aligned}
$$

with $\alpha_{k}=\beta_{k}^{\prime}$ and

$$
\beta_{k}=-\frac{1}{2 k^{2}}\left(h_{k}+6 \eta_{k}\right)
$$

The transformation for the vector field $A_{\mu}$ and its energy-momentum tensor are given by $\delta_{\zeta} A_{\mu}=-\mathcal{L}_{\zeta} A_{\mu}$ and $\delta_{\zeta} T^{\mu}{ }_{\nu}=-\mathcal{L}_{\zeta} T^{\mu}{ }_{\nu}$ respectively. From these transformation laws we obtain for the vector field

$$
\begin{gathered}
\delta_{\zeta} \mathcal{A}_{0}=-\left(\alpha \mathcal{A}_{0}\right)^{\prime} \\
\delta_{\zeta} \mathcal{A}_{i}=-\partial_{i} \alpha \mathcal{A}_{0}
\end{gathered}
$$

so that the perturbations $\delta \mathcal{A}_{0}$ and $\delta \mathcal{A}$ in both gauges relate as:

$$
\begin{aligned}
\delta \mathcal{A}_{0}^{\text {conf }} & =\delta \mathcal{A}_{0}^{\text {syn }}+\left(\alpha A_{0}\right)^{\prime}-2 \mathcal{H} \alpha A_{0} \\
\mathcal{A}^{\text {conf }} & =\mathcal{A}^{\text {syn }}+\alpha A_{0}
\end{aligned}
$$

These relations show that the combination $\delta \mathcal{A}_{0}-\mathcal{A}+2 \mathcal{H} A_{0}$ is gauge-invariant (in the sense of cosmological perturbations).

On the other hand, for the energy-momentum tensor we obtain:

$$
\begin{aligned}
\delta_{\zeta} T_{0}^{0} & =-\alpha T_{0,0}^{0} \\
\delta_{\zeta} T_{j}^{i} & =-\alpha T_{j, 0}^{i} \\
\delta_{\zeta} T_{i}^{0} & =-\left(T_{0}^{0}-\frac{1}{3} T_{j}^{j}\right) \partial_{i} \alpha
\end{aligned}
$$

It is very interesting to note that, given that the background evolution of the vector field is the same as that of a cosmological constant we have that both the unperturbed energy density and pressure are constant and they satisfy $\rho+p=0$ so that the energy momentum tensor remains invariant after the gauge transformation. In fact, this is what one would expect from Stewart-Walker lemma [36]. Notice also that due to the gauge invariance of the energy momentum tensor the results showed in Fig. 11 are also valid for the synchronous gauge.

The perturbed equations for the vector field in the synchronous gauge are:

$$
\begin{aligned}
& \delta \mathcal{A}_{0 k}^{\prime \prime}-4 \mathcal{H} \delta \mathcal{A}_{0 k}^{\prime}-3 k^{2} \delta \mathcal{A}_{0 k}=-2 k^{2}\left(2 \delta \mathcal{A}_{k}^{\prime}-5 \mathcal{H} \delta \mathcal{A}_{k}\right)-\frac{1}{2}\left[\left(h_{k}^{\prime \prime}-4 \mathcal{H} h_{k}^{\prime}\right) A_{0}+h_{k}^{\prime} A_{0}^{\prime}\right] \\
& \delta \mathcal{A}_{k}^{\prime \prime}-4 \mathcal{H} \delta \mathcal{A}_{k}^{\prime}+\left(4 \mathcal{H}^{2}-2 \mathcal{H}^{\prime}-\frac{1}{3} k^{2}\right) \delta \mathcal{A}_{k}=2\left(\frac{2}{3} \delta \mathcal{A}_{0 k}^{\prime}-\mathcal{H} \delta \mathcal{A}_{0 k}\right)+\frac{1}{6} h_{k}^{\prime} A_{0}
\end{aligned}
$$


and the perturbed energy-momentum components in the synchronous gauge are:

$$
\begin{aligned}
\delta T_{0}^{0} & =\frac{1}{6 a^{8}}\left\{\left(2 \delta \mathcal{A}_{0 k}^{\prime}+A_{0} h_{k}^{\prime}\right) A_{0}^{\prime}+2 k^{2}\left[\left(-3 \delta \mathcal{A}_{0 k}+3 \delta \mathcal{A}_{k}^{\prime}-6 \mathcal{H} \delta \mathcal{A}_{k}\right) A_{0}+A_{0}^{\prime} \delta \mathcal{A}_{k}\right]\right\} \\
\delta T^{i}{ }_{j} & \left.=\frac{1}{6 a^{8}}\left\{\left(2 \delta \mathcal{A}_{0 k}^{\prime}+A_{0} h_{k}^{\prime}\right) A_{0}^{\prime}+2 k^{2}\left[3 \delta \mathcal{A}_{0 k}-3 \delta \mathcal{A}_{k}^{\prime}+6 \mathcal{H} \delta \mathcal{A}_{k}\right) A_{0}+A_{0}^{\prime} \delta \mathcal{A}_{k}\right]\right\} \delta^{i}{ }_{j} \\
\delta T_{i}^{0} & =-\frac{i k_{i}}{6 a^{8}} A_{0}\left[h_{k}^{\prime} A_{0}+2 \delta \mathcal{A}_{0 k}^{\prime}+2 k^{2} \delta \mathcal{A}_{k}\right]
\end{aligned}
$$

Condition (25) remains the same in this case:

$$
\delta\left(\rho_{k}+p_{k}\right)=-\frac{2 A_{0} k^{2}}{a^{8}}\left(\delta \mathcal{A}_{0 k}-\delta \mathcal{A}_{k}^{\prime}+2 \mathcal{H} \delta \mathcal{A}_{k}\right)
$$

which is just a consequence of the aforementioned fact that the combination $\delta \mathcal{A}_{0}-\delta \mathcal{A}^{\prime}+2 \mathcal{H} \delta \mathcal{A}$ does not depend on the gauge choice. 